\title{
Survival Analysis in Pediatric Osteosarcoma
}

\author{
Mururul Aisyi ${ }^{1}$, Ayu Hutami Syarif ${ }^{2}$, Nur Asih Anggraeni ${ }^{2}$, Adhitya Bayu Perdana ${ }^{2}$, \\ Hutomo Rezky ${ }^{3}$, Agus Kosasih ${ }^{4}$, Achmad Basuki ${ }^{5}$ \\ ${ }^{1}$ Pediatric Hematology Oncology Department, National Cancer Center-Dharmais Cancer Hospital, Jakarta, Indonesia \\ ${ }^{2}$ Research and Development Department, National Cancer Center-Dharmais Cancer Hospital, Jakarta, Indonesia \\ ${ }^{3}$ General Medicine Department, National Cancer Center-Dharmais Cancer Hospital, Jakarta, Indonesia \\ ${ }^{4}$ Clinical Pathology Department, National Cancer Center-Dharmais Cancer Hospital, Jakarta, Indonesia \\ ${ }^{5}$ Orthopedic Surgery Department, National Cancer Center-Dharmais Cancer Hospital, Jakarta, Indonesia
}

\section{ARTICLE INFO}

Received : 25 January 2019

Reviewed: 10 May 2019

Accepted : 06 June 2019

\section{Keywords:}

osteosarcoma, pediatric, survival

\begin{abstract}
Background: Osteosarcoma is the most common bone malignancy in childhood and adolescence. Despite significant advances in diagnostic and therapeutic modalities, osteosarcoma has generally poor prognosis. Several studies highlighted the prognostic significance of demographic and clinical parameters in the pediatric population. Controversy exists about which obvious factors of mortality in some institutions. Meanwhile, little is known about the overall survival (OS) of pediatric osteosarcoma in Dharmais Cancer Hospital. The aim of this study is to estimate the OS in pediatric osteosarcoma.
\end{abstract}

\begin{abstract}
Methods: This retrospective cohort study enrolled 41 children with osteosarcoma during the period of January 2010-September 2017. Patients were selected using inclusion and exclusion criteria. Overall survival (OS) and patient variables were plotted using Kaplan-Meier.
\end{abstract}

Results: Our result showed that all patients admitted to our hospital in advanced stages (IIB and III). Their median OS was 23 months (12.3-33.7 months) with the survival probability was $29.4 \%$.

Conclusions: Median OS of pediatric osteosarcoma in our institution was 23 months and the survival probability was $29.4 \%$. It can be used as evidence showing lack of cancer awareness and early detection in our country.

\section{INTRODUCTION}

Osteosarcoma is the most common malignancy of the bone which primarily affects primitive bone-forming mesenchymal cells. Osteosarcoma is distributed by age in a bimodal pattern, reaching peaks in adolescence (1014 years) and elderly (older than 65 years) [1]. The adolescent period was characterized by the rapid growth of the long bones and subsequently correlates with the appearance of the tumor [2]. Osteosarcoma may occur in any anatomical location. However, it prefers the metaphyses of long bones such as distal femur, tibia, and proximal humerus. Clinically, the tumors are initially manifested as local pain with or without palpable lump and it occurs over periods of months or years [3].

In the past few decades, there has been a major transformation in the diagnosis and treatment of osteosarcoma and thus improved the survival rate among patients. The 5-year survival rate for the children dramatically increased after the introduction of adjuvant chemotherapy from $10 \%$ to about $60 \%-80 \%$ in localized osteosarcoma and to $15 \%-30 \%$ for metastatic osteosarcoma [4]. Clinical factors associated with prognosis in pediatric osteosarcoma have been described by Enneking thirty years ago. They were the tumor stage and metastasis status [5]. Other recognized prognostic factors were age, gender, tumor site and size, histologic subtype, and elevated Alkaline Phosphatase (ALP) [6].

In addition, inflammation-based score from complete blood count has been explored to predict the prognosis in many cancers, including osteosarcoma. It was known that the increase of neutrophils and decrease of lymphocyte count is the result of systemic inflammatory response. Inflammatory microenvironment promotes angiogenesis and 
metastasis, subverts adaptive immune responses, and alters responses to hormones and chemotherapeutic agents [7]. Due to the association of inflammation in cancer development, the prognostic significance of several inflammation biomarkers and hematological parameters, including neutrophil-lymphocyte ratio (NLR), platelet-lymphocyte ratio (PLR), lymphocytemonocyte ratio (LMR), and neutrophil-platelet score (NPS) have been investigated in osteosarcoma.

Identification of variables which define prognosis allows us to stratify patients into a subgroup with better or worse risk and death due to disease. The aim of this study is to estimate the overall survival (OS) in pediatric osteosarcoma patients treated in Dharmais National Cancer Center.

\section{METHODS}

\section{Patients}

This retrospective cohort study used total sampling which involved 48 children with osteosarcoma during January 2010-September 2017 in Dharmais Cancer Hospital. All new patients aged 2-18 years old were included in the study. We excluded patients with incomplete data (Figure 1).

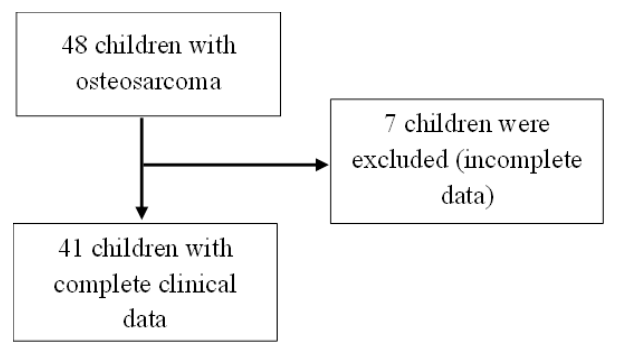

Figure 1. Patient's inclusion flowchart

Demographic and clinical data (age, gender, disease stage, tumor site, the presence of metastasis, complete blood count) were obtained from medical records while time to death data was collected by interviewing relatives by phone in September-October 2017. Follow up time varied from 1 to 72 months. Ethical clearance was approved by the institution's review board.

\section{Variable definition}

Disease stages were grouped as IIB and III based on Enneking Staging System. The presence of metastases was considered as yes or no. The location of tumor was separated into upper long bones and lower long bones. To assess survival outcome, patients' death were categorized as failed or not failed. Neutrophil, lymphocyte, platelet, monocyte count was obtained and calculated to measure the NLR, PLR, LMR, and NPS. The
NLR was derived by dividing the neutrophil count by the lymphocyte count, and separated into high NLR (>2) and low NLR $(\leq 2)$ based on the cut off value; the PLR was derived by dividing the platelet count by the lymphocyte count, and grouped as high PLR $(>150)$ and low PLR $(\leq 150)$; the LMR was obtained by dividing the lymphocyte count by the monocyte count, and categorized into high $\operatorname{LMR}(>3,43)$ and low $\operatorname{LMR}(\leq 3,43)$; and the NPS was resulted from combining the absolute neutrophil count and platelet count, and divided into high NPS (Absolute Neutrophil Count >7.5 and thrombocyte $>400.000$ ), medium NPS (Absolute Neutrophil Count $>7.5$ or thrombocyte $>400.000$ ), and low NPS (Absolute Neutrophil Count $\leq 7.5$ and thrombocyte $>400.000$ ). Overall survival (OS) was defined as the length of time from diagnosis to the occurred event.

\section{Statistical analysis}

Data were analyzed statistically with Statistic Package for Social Service (SPSS) version 22. All parameters were compared to each other in univariate analysis. These variables were expressed in percentages. The OS and patient variables were analyzed by KaplanMeier method by inserting time of death, status of the event, and independent variables.

\section{RESULTS}

\section{Patients characteristics}

A total of 41 children enrolled in this study consisted of 20 males and 21 females with median age of 12 years (8-18 years). About $56.1 \%$ of patients were in stage IIB at the time of diagnosis and $43.9 \%$ were in stage III. Furthermore, lower long bones were the predominant sites for osteosarcoma (68.3\%) and half of the patients (56.1\%) suffered non-metastatic disease. Most of our patients marked in a high ratio of NLR and LMR but less in PLR and NPS ratio (Table 1).

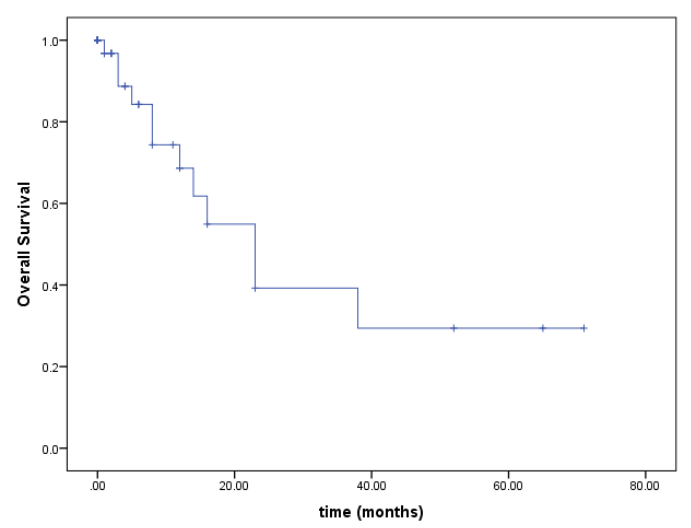

Figure 2. Overall survival children with osteosarcoma 
The median OS of all patients was 23 months (12.333.7 months) and the survival cumulative probability was $29.4 \%$ (Figure 2). All variables showed no statistical difference in OS ( $P>0.05)$ (Table 2).

Table 1. Demographic data

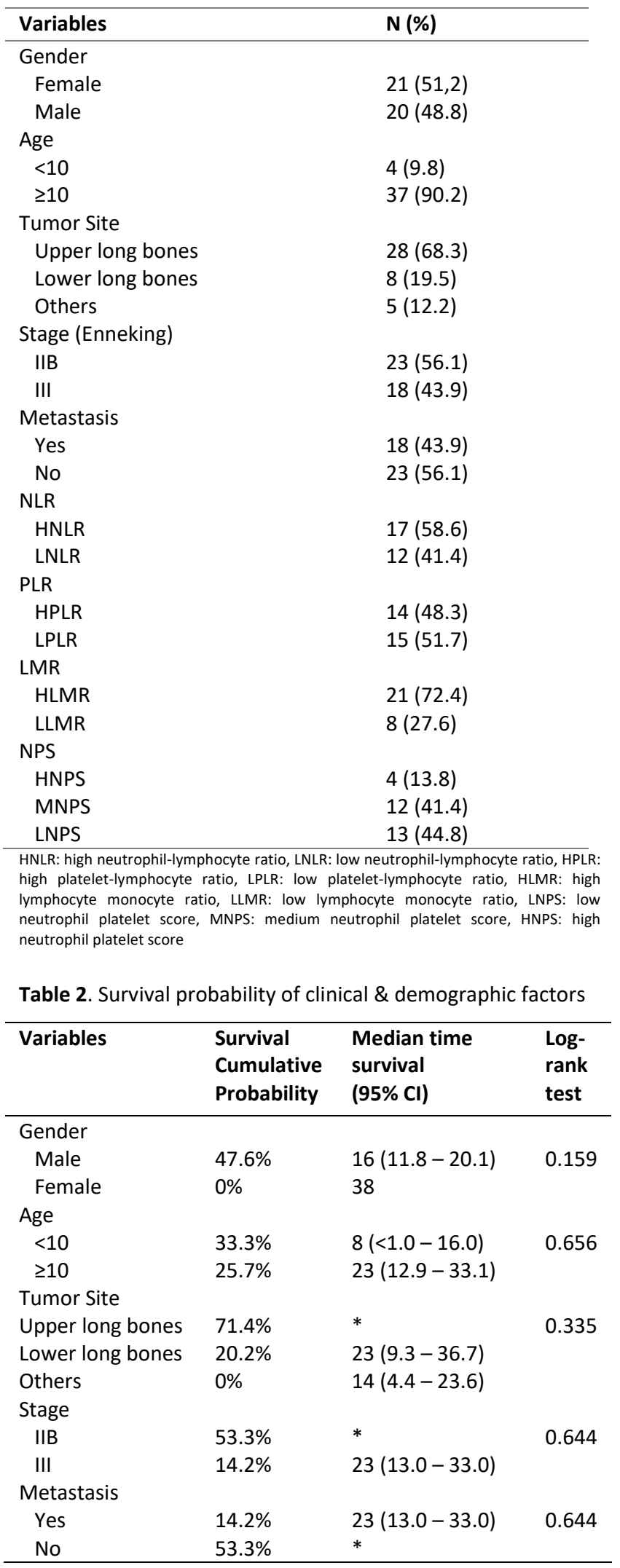

Among 41 patients, only 29 patients who had complete blood count data that were analyzed for survival probability of the inflammatory markers (Table 3).

Table 3. Survival probability of inflammatory markers

\begin{tabular}{|c|c|c|c|}
\hline Variables & $\begin{array}{l}\text { Survival } \\
\text { Cumulative } \\
\text { Probability }\end{array}$ & $\begin{array}{l}\text { Median time } \\
\text { Survival } \\
\text { (months) }\end{array}$ & $\begin{array}{l}\text { Log-rank } \\
\text { test }\end{array}$ \\
\hline \multicolumn{4}{|l|}{ NLR } \\
\hline LNLR & $40.6 \%$ & $23(5.2-40.8)$ & 0.908 \\
\hline HNLR & $31.6 \%$ & $23(<1.0-49.1)$ & \\
\hline \multicolumn{4}{|l|}{ PLR } \\
\hline LPLR & $51.6 \%$ & $*$ & 0.414 \\
\hline HPLR & $18.5 \%$ & $23(8.0-38.0)$ & \\
\hline \multicolumn{4}{|l|}{ LMR } \\
\hline HLMR & $31.5 \%$ & $23(<1.0-52.1)$ & 0.980 \\
\hline LLMR & $40.0 \%$ & $23(<1.0-47.7)$ & \\
\hline \multicolumn{4}{|l|}{ NPS } \\
\hline LNPS & $35.0 \%$ & $23(12.3-33.6)$ & 0.202 \\
\hline MNPS & $45.0 \%$ & $38(7.9-68.0)$ & \\
\hline HNPS & $0 \%$ & 12 & \\
\hline
\end{tabular}

\section{DISCUSSION}

The accuracy of survival evaluation is essential for treating osteosarcoma. The 5-year OS of 41 patients was 23 months with a probability to survive at $29.4 \%$. This finding is not consistent with previous reports from several other countries. A report from the Netherlands evaluating 130 patients had a higher 5 -year OS of $78.6 \%$ [7]. A better result (88.6\%) was released in the US which evaluated 733 patients in a population-based study [8] Other Asian countries such as Malaysia and Thailand included bigger participants and yield higher OS (44\% and $53.2 \%$, respectively) $[9,10]$. These OS gaps were possibly caused by the advanced stage of our osteosarcoma patients admitted to our institution as a tertiary referral center.

Current factors, including age, gender, tumor site, stage, and metastasis, were associated with the survival of osteosarcoma in many studies. Age has been known as prognostic factors for osteosarcoma. Studies found that age was associated with survival of patients [11, 12]. Along with this, a study by Sha et al. [13] reported gender and metastasis as independent prognostic factors in patients with local recurrence.

This present study included a group of 41 patients who were treated at Dharmais Cancer Hospital. Patientrelated variables (age, gender, the presence of metastasis, tumor site, and stage) were assessed and analyzed for their survival probability. Apart from previous studies, our result showed no significant difference in survival probability among those variables. 
Our study reported that all inflammatory markers showed no association with survival. Systemic inflammatory markers have been explored and can be used to determine the prognosis and survival of osteosarcoma patients. As the peripheral blood count test is routinely performed without additional effort, it is simple, reproducible, and inexpensive to measure the response of inflammation and prognosis [14]. Pretreatment NLR was associated with OS in pediatric sarcoma, including osteosarcoma [15]. NPS value in a different type of cancer associated with poorer survival. Post-operation PLR and LMR ratio was still debatable to predict the prognosis $[14,16]$. However, the current study found no association between NLR, PLR, LMR, and NPS ratio with patients' survival.

The result of our study was unable to represent the larger pediatric osteosarcoma population due to its small number of participants. Another study limitation was complete blood count data were not available in some patients.

\section{CONCLUSION}

Median OS of pediatric osteosarcoma in our institution was 23 months and the survival probability was $29.4 \%$. This low survival needs to be addressed by a better management strategy including improving cancer awareness and early detection of osteosarcoma.

\section{DECLARATIONS}

\section{Competing of Interest}

The authors declare no competing interests.

\section{Acknowledgment}

The authors gratefully acknowledge the support from the R\&D Department staff, especially Anita Meisita, SKM, who provided statistical analysis for this study.

\section{REFERENCES}

1. Nedelcu D, Andreescu N, Boeriu E, Stefanescu R, Arghirescu S, Puiu M. Retrospective study on osteosarcoma and ewing sarcoma-our experience. Maedica. 2014;9(2):151-6.

2. Davies AJ. Clinical and molecular prognostic factors in follicular lymphoma. Curr Oncol Rep. 2006;8(5):359-67.

3. Haddox CL, Han G, Anijar L, Binitie O, Letson GD, Bui $\mathrm{MM}$, et al. Osteosarcoma in pediatric patients and young adults: A single institution retrospective review of presentation, therapy, and outcome. Sarcoma. 2014;2014.

4. The American Cancer Society Medical and editorial content team. Survival Rates for Osteosarcoma
[Internet]. The American Cancer Society. 2018. Available from: https://www.cancer.org/cancer/osteosarcoma/det ection-diagnosis-staging/survival-rates.html

5. Enneking WF, Spanier SS, Goodman MA. A system for the surgical staging of musculoskeletal sarcoma. Clin Orthop Relat Res. 2019;(153):106-20.

6. Zamzam MA, Moussa EAHH, Ghoneimy AE, et al. Outcomes and prognostic factors for nonmetastatic osteosarcoma of the extremity. SM Journal of Pediatrics. 2017;2(2).

7. Liu B, Huang Y, Sun Y, Zhang J, Yao Y, Shen Z, et al. Prognostic value of inflammation-based scores in patients with osteosarcoma. Sci Rep. 2016;6(600):1-9.

8. Hagleitner MM, De Bont ESJM, Maroeska D, Te Loo WM. Survival trends and long-term toxicity in pediatric patients with osteosarcoma. Sarcoma. 2012;2012.

9. Nagarajan R, Kamruzzaman A, Ness KK, Marchese VG, Sklar C, Mertens A, et al. Twenty years of follow-up of survivors of childhood osteosarcoma: A report from the childhood cancer survivor study (CCSS). Cancer. 2011;117(3):625-34.

10. Choeyprasert W, Pakakasama S, Sirachainan N, Songdej D, Chuansumrit A, Anurathapan $U$, et al. Comparative outcome of Thai pediatric osteosarcoma treated with two Protocols: the role of high-dose methotrexate (HDMTX) in a single institute experience. Asian Pacific J Cancer Prev. 2014;15:9823-7.

11. Faisham WI, Mat Saad AZ, Alsaigh LN, Nor Azman MZ, Kamarul Imran M, Biswal BM, et al. Prognostic factors and survival rate of osteosarcoma: A singleinstitution study. Asia Pac J Clin Oncol. 2017;13(2):e104-10.

12. Maza I, Vasquez L, Geronimo J, Silva JM, Sialer L, Tarrillo $F$, et al. Analysis of prognostic factors in high-grade osteosarcoma of the extremities in children: A 15-year single-institution experience. Front Oncol. 2016;6:6-11.

13. Sha J, Qi W, Hu H, Sun $Y$, Shen Z, Yao $Y$. Retrospective analysis of prognostic factors for sixty osteosarcoma patients with local recurrence. 2013.

14. Liu T, Fang XC, Ding Z, Sun ZG, Sun LM, Wang YL. Pre-operative lymphocyte-to-monocyte ratio as a predictor of overall survival in patients suffering from osteosarcoma. FEBS Open Bio. 2015;5:682-7.

15. Vasquez L, León E, Beltran B, Maza I, Oscanoa M, Geronimo J. Pretreatment neutrophil-tolymphocyte ratio and lymphocyte recovery. J Pediatr Hematol Oncol. 2017;00(00):1.

16. Xia WK, Liu ZL, Shen D, Lin QF, Su J, Mao WD. Prognostic performance of pre-treatment NLR and PLR in patients suffering from osteosarcoma. World J Surg Oncol. 2016;14(1):1-8. 